\title{
Determination of vanillin, ethyl vanillin, and coumarin in infant formula by liquid chromatography-quadrupole linear ion trap mass spectrometry
}

\author{
Yan Shen, ${ }^{\star 1}$ Chao Han, † Bin Liu, † Zhengfeng Lin, $¥$ Xiujin Zhou,§ Chengjun Wang, ${ }^{*}$ and Zhenou Zhu† \\ ${ }^{*}$ College of Chemistry and Materials Engineering, Wenzhou University, Wenzhou 325035, China \\ †Wenzhou Entry-Exit Inspection and Quarantine Bureau of P.R.C, Wenzhou 325027, China \\ †Hainan Entry-Exit Inspection and Quarantine Bureau of P.R.C, Haikou 570311, China \\ §Zhoushan Entry-Exit Inspection and Quarantine Bureau of P.R.C, Zhoushan 316000, China
}

\begin{abstract}
A simple, precise, accurate, and validated liquid chromatography-quadrupole linear ion trap mass spectrometry method was developed for the determination of vanillin, ethyl vanillin, and coumarin in infant formula samples. Following ultrasonic extraction with methanol/water (1:1, vol/vol), and clean-up on an HLB solid-phase extraction cartridge (Waters Corp., Milford, MA), samples were separated on a Waters XSelect HSS T3 column $(150 \times 2.1-\mathrm{mm}$ i.d., $5-\mu \mathrm{m}$ film thickness; Waters Corp.), with $0.1 \%$ formic acid solution-acetonitrile as mobile phase at a flow rate of 0.25 $\mathrm{mL} / \mathrm{min}$. Quantification of the target was performed by the internal standard approach, using isotopically labeled compounds for each chemical group, to correct matrix effects. Data acquisition was carried out in multiple reaction monitoring transitions mode, monitoring 2 multiple reaction monitoring transitions to ensure an accurate identification of target compounds in the samples. Additional identification and confirmation of target compounds were performed using the enhanced product ion modus of the linear ion trap. The novel liquid chromatography-quadrupole linear ion trap mass spectrometry platform offers the best sensitivity and specificity for characterization and quantitative determination of vanillin, ethyl vanillin, and coumarin in infant formula and fulfills the quality criteria for routine laboratory application.
\end{abstract}

Key words: vanillin, ethyl vanillin, coumarin, infant formula

\section{INTRODUCTION}

Proper nutrition is particularly critical during infancy when growth and development are most rapid and, therefore, the consequences of inadequate nutrition are most severe. Breast milk is the most adequate food for

Received July 27, 2013.

Accepted October 31, 2013.

${ }^{1}$ Corresponding author: shenyan@wzu.edu.cn babies, but in some cases it is not sufficient to feed the infant and infant formula use is imperative (Delange and West, 2003; Sola-Larrañaga and Navarro-Blasco, 2009). Likewise, infant formula is manufactured by modifying cow milk composition so as to resemble that of human milk. It provides all nutrient needs, such as protein, carbohydrates, and lipids.

Vanilla extract is one of the most widely used flavoring ingredients in foods and beverages. Although authentic vanilla extract is thought to provide the most desirable flavor characteristics, the supply of vanilla beans is limited and prices are high. Because of the high cost of authentic vanilla extracts, artificial vanilla flavorings are often used. Artificial vanilla flavorings usually contain synthetically produced vanillin and (or) ethyl vanillin dissolved in alcohol, propylene glycol, and (or) glycerin. Some vanilla extract manufacturers have adulterated vanilla extracts with coumarin to increase the vanilla flavor perception. Coumarin, a phytochemical found in many plant species, has a sweet herbaceous odor and has been used in food, tobacco, and cosmetics as a flavoring and fragrance enhancer. Coumarin has been shown to cause hepatoxicity in animals and has been banned for use as a food additive in the United States since 1956 (Hazleton et al., 1956).

Coumarin was first suspected to have genotoxic and carcinogenic effects in the 1980s (AFC, 2004; Sproll et al., 2008). On this basis, the Codex Alimentarius provided general requirements for natural flavor that included specific maximum levels for coumarin in the final product ready for consumption (Codex Alimentarius, 1985). It must be noted that coumarin must not be added as such to food and beverages. It may only be contributed through the use of natural flavor, provided that the maximum levels in the final product ready for consumption are not exceeded. The Codex Alimentarius maximum levels were subsequently introduced into European law in 1988 (European Council, 1988).

A survey of infant formula and milk was conducted in China. The study found that vanillin or coumarin, or both, was present in some of the products. Several methods have been developed for analysis of vanillin, 
ethyl vanillin, and coumarin; these include HPLC-UV detection (Lavine et al., 2012), gas chromatography or GC-MS (Rahim et al., 2011), liquid chromatographymass spectrometry (LC-MS; de Jager et al., 2007; Rychlik, 2008), and capillary electrophoresis (Ohashi et al., 2007).

Currently, the application of advanced liquid chromatography-tandem mass spectrometry (LC-MS/ MS) to residue analyses has allowed a broad range of compounds to be determined and this has permitted the comprehensive assessment of food contaminants. Low maximum residue limits have fostered the development of more powerful and sensitive analytical methods to meet the requirements of complex samples, such as food. In this sense, LC-MS/MS with a triple quadrupole in multiple reaction monitoring (MRM) mode has become the most widely used technique for the quantitation of residues in food, as reported extensively in the literature (Xie et al., 2011)

Herein, a reliable analytical method of liquid chromatography-quadrupole linear ion trap mass spectrometry (LC-QqLIT-MS) is presented, for the detection of vanillin, ethyl vanillin, and coumarin in infant formula samples. The described method requires a simple sample preparation step and provides a rapid, sensitive, and accurate process to screen for vanillin, ethyl vanillin, and coumarin in infant formula samples.

\section{MATERIALS AND METHODS}

\section{Chemicals, Reagents, and Equipment}

All reagents and solvents were analytical grade unless otherwise specified. Certified standards of vanillin (99.9\% purity) and ethyl vanillin (97.3\% purity) were purchased from ChromaDex (Irvine, CA). Coumarin (98\% purity) was purchased from Dr. Ehrenstorfer $\mathrm{GmbH}$ (Augsburg, Germany). Vanillin- ${ }^{13} \mathrm{C}_{6}$ (99\%) and coumarin- $\mathrm{D}_{4}(98 \%)$ were purchased from Toronto Research Chemicals Inc. (North York, ON, Canada). The structures of these standards are shown in Figure 1. The water used was purified with a Milli-Q water purification system from Millipore Corp. (Bedford, MA). High performance liquid chromatography-grade acetonitrile and methanol were obtained from Merck KGaA (Darmstadt, Germany). Ammonium acetate and formic acid were acquired from Alfa Aesar (Ward Hill, MA). An ultrasonic extraction system (Elma P300 H; Elma Hans Schmidbauer GmbH \& Co. KG, Singen, Germany) and LC-QqLIT-MS (Agilent 1200-API 4000 QTRAP; Agilent Technologies Deutschland GmbH, Böblingen, Germany) were used in sample analysis. The LC and MS/MS analytical conditions are given in Tables 1 and 2 .

\section{Preparation of Standards}

Standard stock solutions (200 mg/L) were prepared in methanol/water $(1: 1, \mathrm{vol} / \mathrm{vol})$ and stored at $4^{\circ} \mathrm{C}$ in the dark. Matrix-matched calibration standards were prepared by adding appropriate volumes of the standard working solution to infant formula extracts and blank samples. Vanillin, ethyl vanillin, and coumarin calibration mixed standards were prepared at concentrations of 1.0, 2.0, 5.0,10.0, 20.0, and $50 \mathrm{ng} / \mathrm{mL}$ before use. We pipetted $100 \mu \mathrm{L}$ of mixed standards $(10,20,50,100$, 200 , and $500 \mathrm{ng} / \mathrm{mL}$; the vanillin $-{ }^{13} \mathrm{C}_{6}$ and coumarin$\mathrm{D}_{4}$ internal standards concentration was $100 \mathrm{ng} / \mathrm{mL}$ ) and then added them to $900-\mu \mathrm{L}$ solid-phase extractions (SPE) of infant formula blank samples, respectively. The vanillin $-{ }^{13} \mathrm{C}_{6}$ and coumarin- $\mathrm{D}_{4}$ internal standards were used to ensure accuracy of the MS response and were added to mixed standards to give a final concentration of $10 \mathrm{ng} / \mathrm{mL}$.

\section{Sample Preparation}

An accurately weighed 1.00-g infant formula sample was placed into a $50-\mathrm{mL}$ polypropylene centrifuge tube. Then, $500 \mu \mathrm{L}$ of a mixed solution of vanillin- ${ }^{13} \mathrm{C}_{6}(400$ $\mathrm{ng} / \mathrm{mL})$ and coumarin- $\mathrm{D}_{4}(400 \mathrm{ng} / \mathrm{mL})$, and $25 \mathrm{~mL}$ of methanol/water $(1: 1, \mathrm{vol} / \mathrm{vol})$ were placed into the centrifuge tube and the tube was capped. The sample was shaken vigorously for $30 \mathrm{~s}$, vortex mixed for $1 \mathrm{~min}$, and then placed in an ultrasonic bath for $30 \mathrm{~min}$. The extraction temperature was set at $30^{\circ} \mathrm{C}$. After extraction, the suspension was centrifuged at $8,875 \times g$ for 15 min. The supernatant layer was then transferred into a $50-\mathrm{mL}$ volumetric flask and diluted with pure water. The extracts were then forwarded to the SPE procedure. Blanks were periodically run during the analysis to confirm the absence of contamination.

\section{SPE}

An Oasis HLB SPE cartridge (60 mg, $3 \mathrm{~mL}$; Waters Corp., Milford, MA) was used to clean up sample extracts. The SPE cartridge was conditioned with methanol $(5 \mathrm{~mL})$ and then with water $(5 \mathrm{~mL})$. The sample $(5 \mathrm{~mL})$ was applied to the conditioned cartridge and allowed to elute by gravity. The cartridge was washed with $5 \mathrm{~mL}$ of water and then with $5 \mathrm{~mL}$ of methanol/ water $(1: 9, \mathrm{vol} / \mathrm{vol})$. The cartridge was dried by applying vacuum for $1 \mathrm{~min}$. The column was eluted into a glass culture tube using $6 \mathrm{~mL}$ of methanol; the eluate was evaporated to dryness under vacuum at $45^{\circ} \mathrm{C}$. The dried extract was reconstituted in $2.0 \mathrm{~mL}$ of methanol/ water $(1: 1, \mathrm{vol} / \mathrm{vol})$, vortex mixed for $60 \mathrm{~s}$, and filtered through a $0.22-\mu \mathrm{m}$ filter in to a glass LC vial. 
Table 1. Analytical conditions for liquid chromatography

\begin{tabular}{ll}
\hline Item & Details \\
\hline Instrument & Agilent 1200 system (Agilent Technologies Deutschland GmbH, Böblingen, Germany) \\
Column & Waters XSelect HSS T3 column $(150 \times 2.1$-mm i.d., 3.5- $\mu$ m film thickness; Waters Corp., Milford, MA) \\
Mobile phase & $\mathrm{A}=0.1 \%$ formic acid; B = acetonitrile; gradient elution used with the ratio of A:B varied as follows: 0 min, 20:80; 1 \\
& $\min , 10: 90 ; 10 \mathrm{~min}, 10: 90 ; 10.1 \mathrm{~min}, 20: 80 ; 15 \mathrm{~min}, 20: 80$. Flow was diverted to waste from 0 to 4.5 min and from 7.5 \\
& to $15 \mathrm{~min}$ \\
Flow rate & $0.25 \mathrm{~mL} / \mathrm{min}$ \\
Column temperature & $30^{\circ} \mathrm{C}$ \\
\hline
\end{tabular}

\section{RESULTS AND DISCUSSION}

\section{Optimization of MS Parameters and MRM Transitions}

Preliminary experiments were conducted for the purpose of finding the best instrumental conditions that would allow unambiguous identification of the analytes in real samples at trace levels. Single vanillin, ethyl vanillin, coumarin, vanillin- ${ }^{13} \mathrm{C}_{6}$, and coumarin- $\mathrm{D}_{4}$ compound standard solutions $(200 \mathrm{ng} / \mathrm{mL})$, prepared in methanol/water $(1: 1, \mathrm{vol} / \mathrm{vol})$, and were introduced into the mass spectrophotometer at a flow rate of 10 $\mu \mathrm{L} /$ min using a syringe pump (Harvard Apparatus Pty Ltd., Brisbane, Australia).
Identification of the parent ion and the choice of the ionization mode for vanillin, ethyl vanillin, coumarin, vanillin- ${ }^{13} \mathrm{C}_{6}$, and coumarin- $\mathrm{D}_{4}$ were performed in the full-scan mode by recording mass spectra from $\mathrm{m} / z 50$ to 200 in positive mode. The most sensitive transition in MRM mode was selected for quantification in the screening method. A minimum of 3 identification points are required to meet the identification performance criteria defined by the European Union Commission for quantitative mass spectrometric detection. Using LC-MS/MS to monitor 1 precursor ion and 2 daughter ions earns 4 identification points ( 1 for the parent ion and 1.5 for each daughter ion) and, therefore, fulfils these criteria.

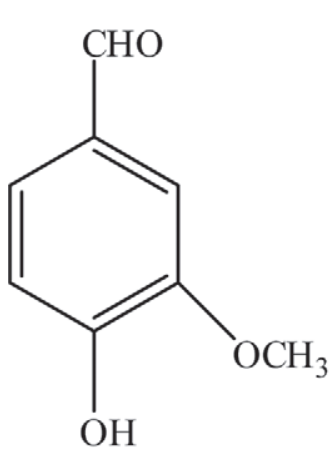

(A)

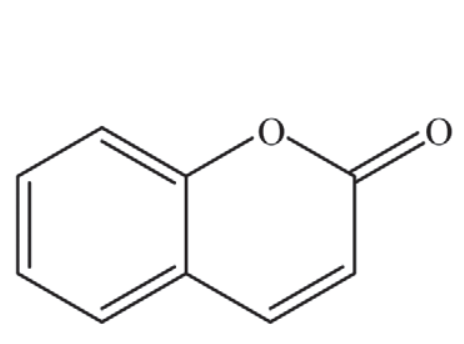

(D)<smiles>CO[14c]1[14cH][14cH][14cH][14c](O)[14cH]1</smiles>

(B)

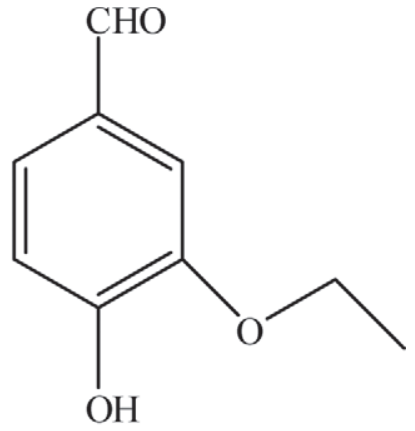

(C)

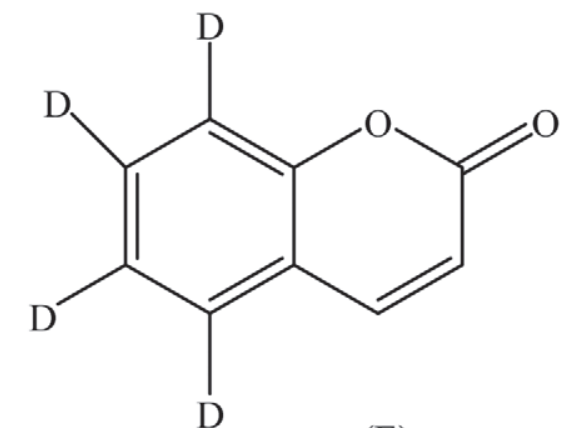

(E)

Figure 1. Chemical structure of vanillin $(A)$, vanillin $-{ }^{13} \mathrm{C}_{6}(\mathrm{~B})$, ethyl vanillin $(\mathrm{C})$, coumarin $(\mathrm{D})$, and coumarin- $\mathrm{D}_{4}(\mathrm{E})$. 
Table 2. Analytical conditions for tandem mass spectrometry (MS/MS; API 4000 QTRAP; Applied Biosystems, Burlington, ON, Canada)

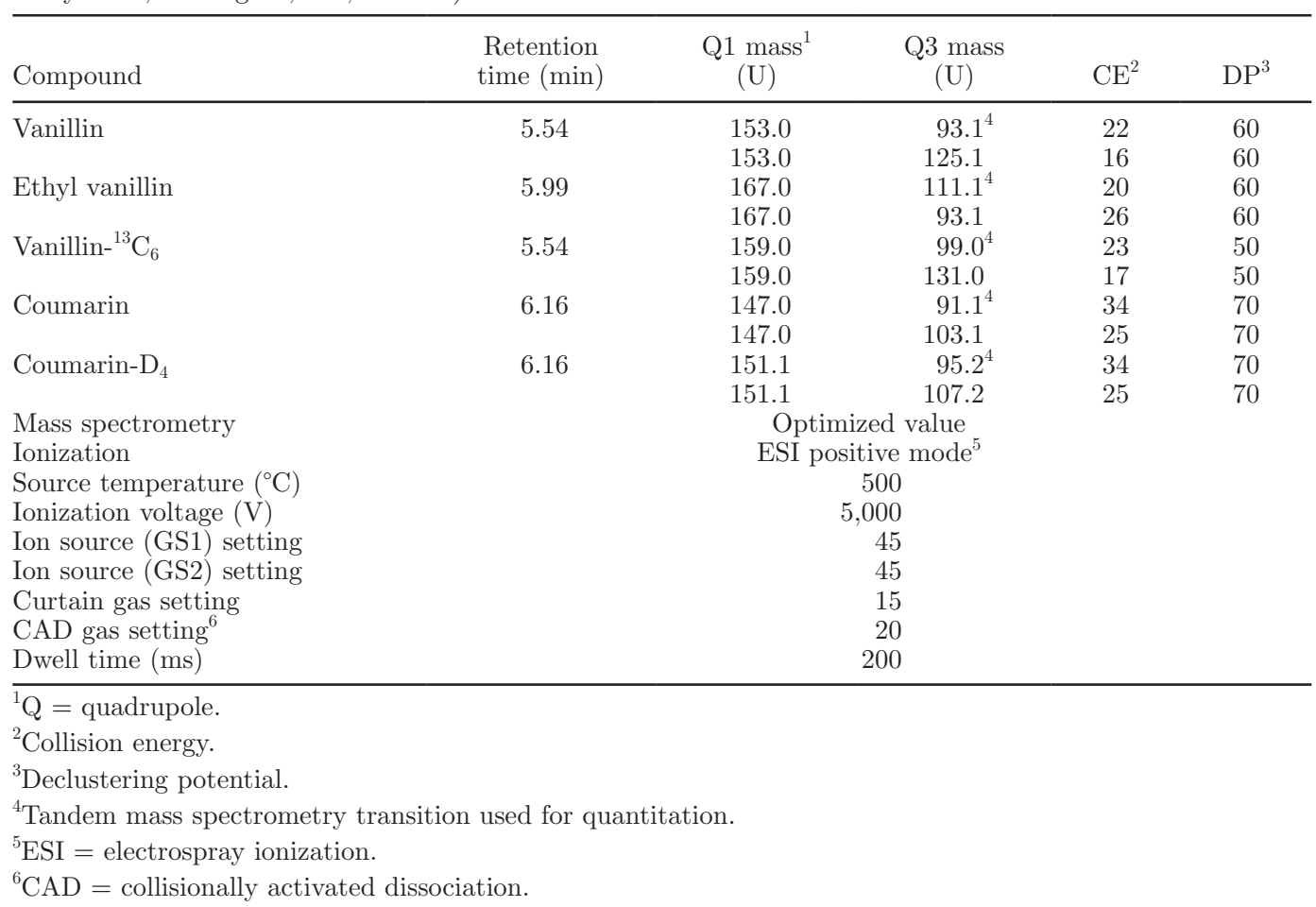

To choose the transitions in the MRM mode, different parameters were studied. The precursor and the product ions of vanillin, ethyl vanillin, coumarin, vanillin- ${ }^{13} \mathrm{C}_{6}$, and coumarin- $\mathrm{D}_{4}$ were selected. The last parameter optimized was the collision energy and declustering potential; different values were tested (15$100 \mathrm{~V})$. In Table 2, we summarize the optimum values for each condition for vanillin, ethyl vanillin, coumarin, vanillin- ${ }^{13} \mathrm{C}_{6}$, and coumarin- $\mathrm{D}_{4}$ compounds. The optimization was done following the normal optimization procedure. In this work, the most intense characteristic MRM transitions were chosen, and Table 2 lists the precursor and daughter ions monitored.

\section{Optimization of Chromatographic Conditions}

To improve vanillin, ethyl vanillin, and coumarin chromatographic separation, different columns and mobile phases were investigated in this study. The results indicated that for the columns investigated, the Waters XSelect HSS T3 column (Waters Corp.) was the optimal one. To enhance the signal response, mobile phase modifiers, such as ammonium acetate, acetic acid, and formic acid, are also discussed. Use of $0.1 \%$ formic acid solution-acetonitrile was found to provide the maximum response and was used in the following method development. As shown in Figure 2, MRM chromatograms of all ions monitored from a spiked sample were satis- factory, except for ethyl vanillin and coumarin, which were close. However, they could be easily identified and quantified in MRM mode due to different parent ions and fragment ions. The results also suggest that the

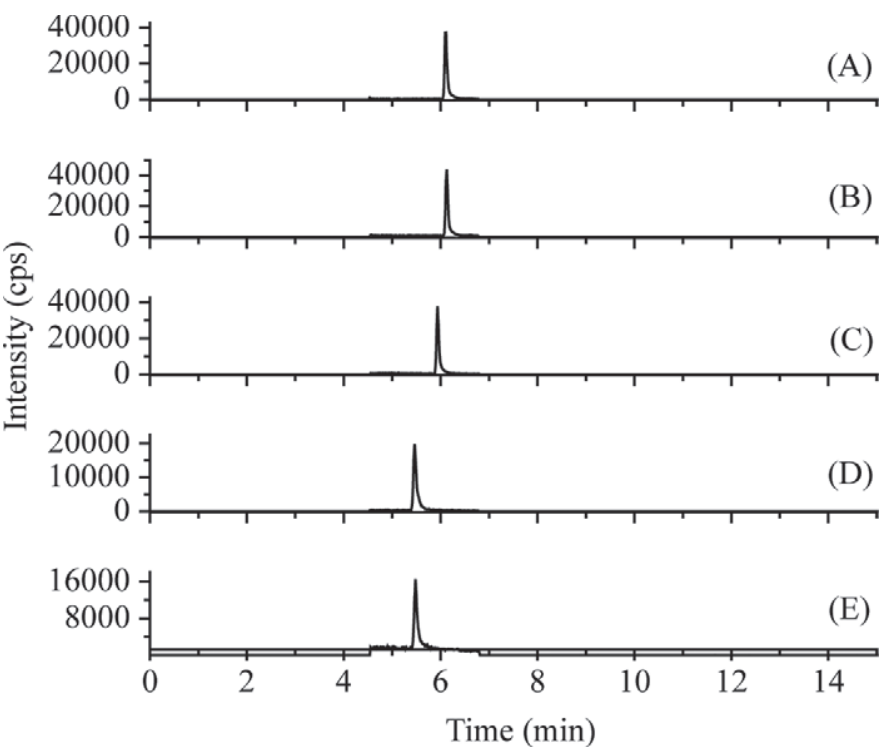

Figure 2. Multiple reaction monitoring (MRM) chromatograms of spiked samples containing coumarin (A), coumarin- $\mathrm{D}_{4}(\mathrm{~B})$, ethyl vanillin $(C)$, vanillin- ${ }^{13} \mathrm{C}_{6}(\mathrm{D})$, and vanillin $(\mathrm{E})$. 
Table 3. Infant formula matrix solution affected selected ions

\begin{tabular}{lcc}
\hline & \multicolumn{2}{c}{ Recovery $(\%)$} \\
\cline { 2 - 3 } Compound & $\begin{array}{c}\text { Solvent } \\
\text { calibration curve }\end{array}$ & $\begin{array}{c}\text { Matrix solution } \\
\text { calibration curve }\end{array}$ \\
\hline Vanillin & 55.4 & 93.2 \\
Ethyl vanillin & 60.3 & 94.6 \\
Coumarin & 68.7 & 103.7 \\
\hline
\end{tabular}

sufficient baseline chromatographic separation has the benefit of reducing the matrix effect.

\section{Matrix Effects}

Matrix effects (generally recognized as a suppression or enhancement of the analytical signal due to coeluting matrix components) have been widely studied and recognized as a source of error in quantitative LC-MS/ MS analysis of food samples (Niessen et al., 2006). In the current study, we chose to use vanillin- ${ }^{13} \mathrm{C}_{6}$ and coumarin- $\mathrm{D}_{4}$ as internal standards to account and correct for matrix effects. The use of matrix-matched calibration standards was done to compensate for the matrix effect (i.e., signal suppression or enhancement of studied compounds in the matrix solution). The matrix effect, expressed as the signal from the vanillin, ethyl vanillin, and coumarin in the matrix compared with the signal in solvent was tested in infant formula sample matrices. Vanillin, ethyl vanillin, and coumarin were significantly affected by the matrix components and the recoveries calculated by the solvent calibration curve were 55.4 to $68.7 \%$. In contrast, the recoveries calculated by the matrix solution calibration curve were 93.2 to $103.7 \%$. Table 3 shows the matrix effects for vanillin, ethyl vanillin, and coumarin at the concentration level of $50 \mu \mathrm{g} / \mathrm{kg}$ in infant formula samples. Recoveries from the matrix solution calibration curve were 35.0 to $37.8 \%$ higher than recoveries from solvent calibration curve. Therefore, for accurate quantification, the use of matrix solution calibration curve is required.

\section{Method Validation}

Linearity of Calibration Standards. The concentrations of the analytes in the samples were calculated by matrix calibration using internal standards. It was evident from the results that reproducible results were obtained using vanillin- ${ }^{13} \mathrm{C}_{6}$ and coumarin- $\mathrm{D}_{4}$ as the internal standard for quantification of the analyzed substances. Calibration curves with $1 / x$ weighting were plotted for each individual analyte. The matrix calibration curve was derived using a series of standards ranging from 1 to $50 \mathrm{ng} / \mathrm{mL}$ and fixed concentrations $(10 \mathrm{ng} / \mathrm{mL})$ of the internal standards. The response data were adjusted according to the relative ratios of the responses to the stable isotope internal standards in the sample and the standard. The correlation coefficients were between 0.9993 and 0.9996. The instrumental limit of detection was calculated as 3 times the standard deviation; it was $3.0,1.5$, and $1.5 \mu \mathrm{g} / \mathrm{kg}$ for vanillin, ethyl vanillin, and coumarin, respectively. The limit of quantification was calculated as 10 times the standard deviation; it was $10.0,5.0$, and $5.0 \mu \mathrm{g} / \mathrm{kg}$ for vanillin, ethyl vanillin, and coumarin, respectively. Quantification was performed based on calibration plots using the peak area of the most intense transition of the analyte.

Assay Specificity. The specificity was evaluated by the analysis of 10 blank samples of different infant formula samples. No interfering peaks from endogenous compounds were found in the retention time of the target analyte for infant formula samples.

Precision and Accuracy. Intraday precision was examined by analysis of a spiked sample at 3 different concentrations (high, medium, and low) on the same day; interday precision was determined by analysis of a spiked sample at 3 different concentrations (high, medium, and low) on 3 different days. Relative standard deviation values were 4.36 and $4.52 \%$, respectively. Method accuracy was evaluated by recovery studies, using "blank" infant formula samples labeled as or-

Table 4. Mean recoveries and repeatability of the developed method at 3 concentration levels with limit of quantification (LOQ; $\mathrm{n}=6$ )

\begin{tabular}{lcccc}
\hline Compound & $\begin{array}{c}\text { Spiking level } \\
(\mu \mathrm{g} / \mathrm{kg})\end{array}$ & $\begin{array}{c}\text { Recovery } \\
(\%)\end{array}$ & $\begin{array}{c}\mathrm{RSD}^{1} \\
(\%)\end{array}$ & $\begin{array}{c}\mathrm{LOQ} \\
(\mu \mathrm{g} / \mathrm{kg})\end{array}$ \\
\hline Vanillin & 10 & 85.4 & 4.5 & \\
& 50 & 90.3 & 3.8 & 10 \\
Ethyl vanillin & 100 & 95.2 & 3.3 & 5 \\
& 10 & 89.8 & 4.3 & \\
Coumarin & 50 & 93.7 & 3.6 & 5 \\
& 100 & 95.6 & 3.0 & 5.2 \\
\end{tabular}

${ }^{1}$ Relative SD. 

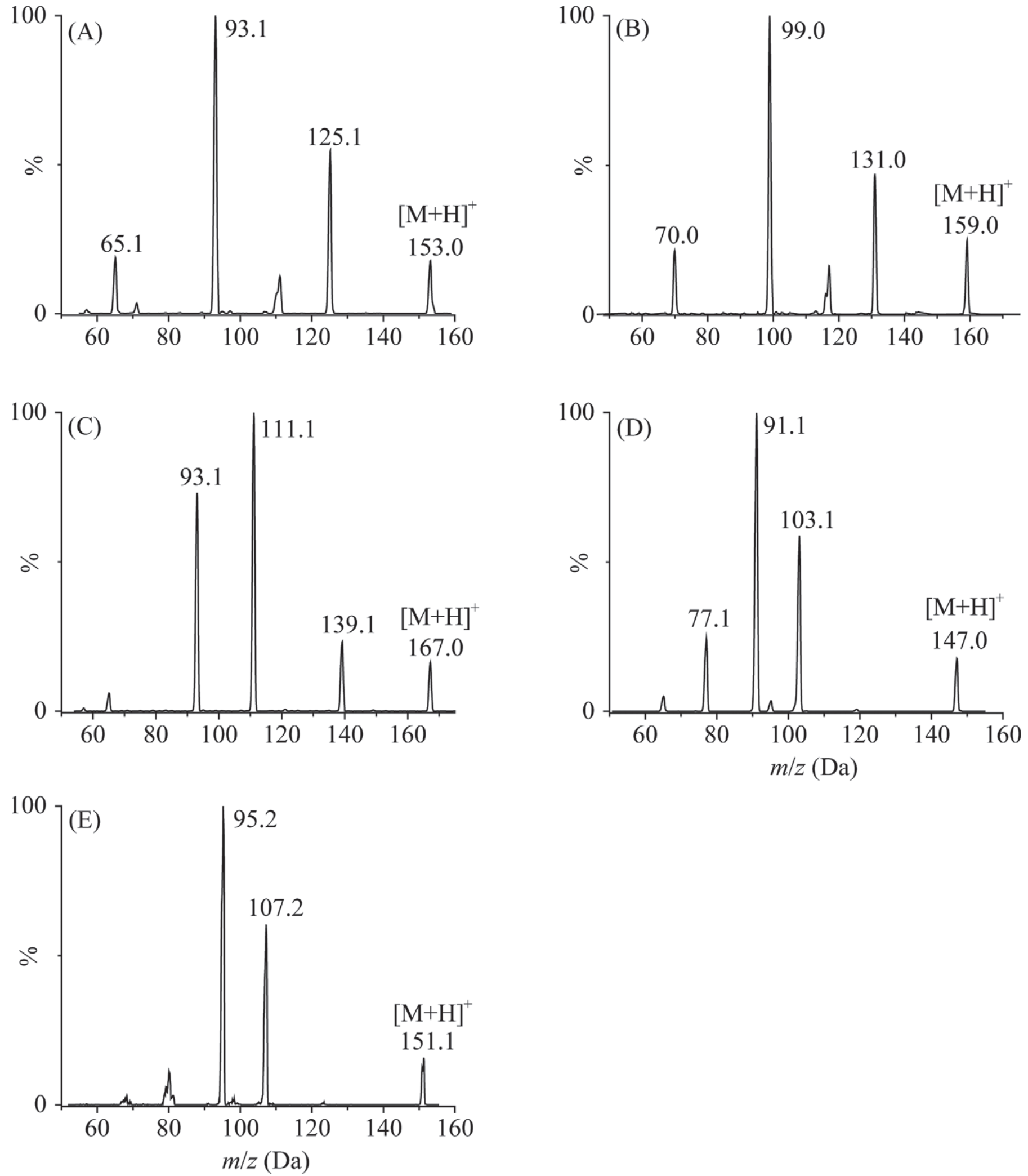

Figure 3. Corresponding enhanced product ion spectrum of vanillin (A), vanillin ${ }^{13} \mathrm{C}_{6}(\mathrm{~B})$, ethyl vanillin $(\mathrm{C})$, coumarin $(\mathrm{D})$, and coumarin- $\mathrm{D}_{4}$ (E). $m / z=$ mass-to-charge ratio. 
(A)

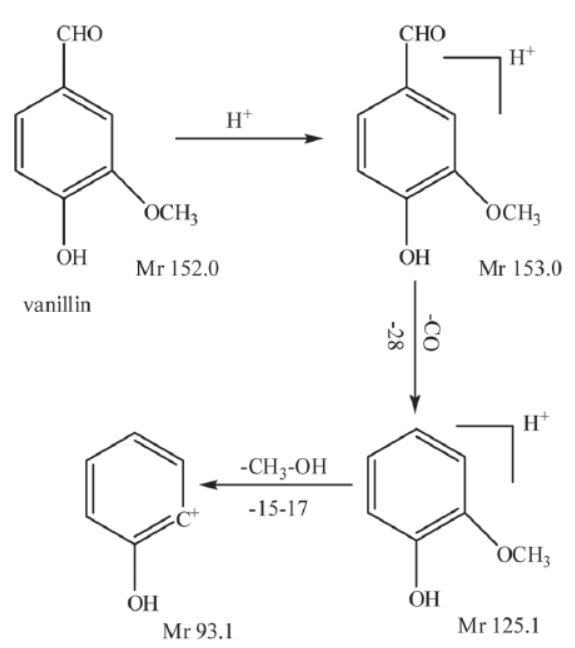

(B)

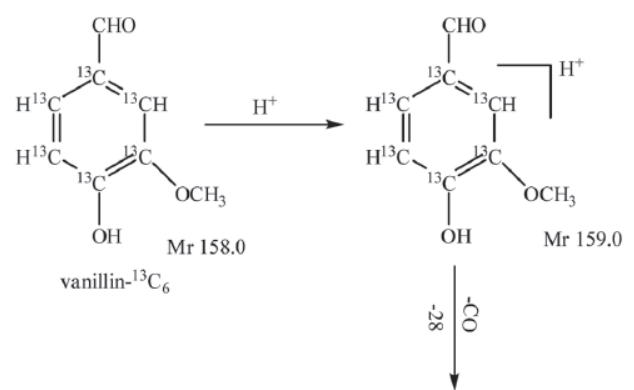<smiles>C[I+]c1[12cH][13cH][13c](O)[14c](O)[14c]1O</smiles>

(C)<smiles>CCOc1cc(C=O)ccc1O</smiles>
ethyl vanillin<smiles>C=Cc1ccccc1O</smiles>
Mr 93.1<smiles>CCOc1cc(C=O)ccc1O</smiles>

(E)<smiles>[2H]c1c([2H])c([2H])c2oc(=O)ccc2c1[18O][I-]([2H])c1c([2H])c([2H])c2oc(=O)ccc2c1[2H]</smiles>
Coumarin-D4<smiles>CC(C)[Mg]</smiles><smiles>[2H]c1cc(Cc2c([2H])c([2H])c([2H])c([2H])c2[2H])c([2H])c([2H])c1[2H]</smiles><smiles>C[In]c1ccc2oc(=O)ccc2c1</smiles>

Mr 147.1<smiles>C[I+]1Cc2ccccc2C1Cc1cccc(Cc2ccccc2)c1</smiles>

Mr 91.<smiles>C[As](C)=O</smiles>

Mr 103.1 
ganically produced and confirmed as not containing the target analytes using the proposed method. "Blank" infant formula samples were spiked with the appropriate amounts of the target compounds at 3 concentration levels: 10, 50, and $100 \mu \mathrm{g} / \mathrm{kg}$. The target analytes contents were determined by use of the corresponding calibration plot and recovery was calculated from the ratio of the amounts detected to those added. Average recovery was in the range 85.4 to $104.8 \%$ (Table 4) and, with relative standard deviations below $4.5 \%$, indicative of the good recovery and precision of the method.

Real Samples. Initial chromatographic experiments were carried out to verify the possibility of proposing an LC-QqLIT-MS method for the determination of vanillin, ethyl vanillin, and coumarin. Initial extraction experiments using vanillin-, ethyl vanillin-, and coumarin-spiked samples demonstrated the selectivity of the method. Figure 2 shows an MRM chromatogram corresponding to 1 spiked sample, where a clean background was obtained, demonstrating the absence of chromatographic interferents. The corresponding enhanced product ion spectra of vanillin, vanillin- ${ }^{13} \mathrm{C}_{6}$, ethyl vanillin, coumarin, and coumarin- $\mathrm{D}_{4}$ are shown in Figure 3A-E. The most plausible interpretation of MS/MS fragmentation was given in Figure 4, primarily based on the enhanced product ion spectra information in Figure 3; currently, no related reported work is available as a reference. Twenty infant formula samples were purchased from local supermarkets and analyzed following the proposed method; vanillin was detected at concentration levels ranging from 2.3 to $712.4 \mathrm{mg} /$ $\mathrm{kg}$ in 4 samples.

\section{CONCLUSIONS}

A rapid and efficient method for simultaneous identification and quantification of vanillin, ethyl vanillin, and coumarin in infant formula samples by LC-QqLIT-MS was established, which can facilitate convenient and rapid quality control of infant formula samples. The coupling of LC separation with MS/MS provides an attractive tool for the identification of vanillin, ethyl vanillin, and coumarin in infant formula samples. Moreover, the availability of the linear ion trap fragment patterns for each analyte enables a comfortable component confirmation in complex samples.

\section{ACKNOWLEDGMENTS}

Financial support from the Science Technology Department of Zhejiang Province (China; 2013C37065),
Certification and Accreditation Administration (CNCA, Beijing, China; 2008B169), The Ministry of Science and Technology (MOST, Beijing, China; 201410070), and National Natural Science Foundation (NSFC, Beijing, China; 21207102) is gratefully acknowledged.

\section{REFERENCES}

AFC. 2004. Opinion of the Scientific Panel on Food Additives, Flavourings, Processing Aids and Materials in Contact with Food (AFC) on a request from the Commission related to coumarin. EFSA J. 104:1-36.

Codex Alimentarius. 1985. General requirements for natural flavourings (CAC/GL 29.1987). Accessed Nov. 28, 2007. http://www. codexalimentarius.org/search-results/?cx $=01817062014370110493$ $3 \% 3 \mathrm{Ai}$-zresgmxec \&cof $=$ FORID $\% 3 \mathrm{~A} 11 \& \mathrm{q}=\mathrm{Codex}+$ alimentarius + 1985 . +coumarin $+\&$ siteurl $=\mathrm{http} \% 3 \mathrm{~A} \% 2 \mathrm{~F} \% 2 \mathrm{Fwww}$.codexalimentarius.org\%2F\&sa. $x=21 \&$ sa. $y=8$.

de Jager, L. S., G. A. Perfetti, and G. W. Diachenko. 2007. Determination of coumarin, vanillin, and ethyl vanillin in vanilla extract products: Liquid chromatography mass spectrometry method development and validation studies. J. Chromatogr. A 1145:83-88.

Delange, F. M., and K. P. West Jr. 2003. Micronutrient Deficiencies in the First Months of Life. Nestlé Nutrition Workshop Series Pediatric Program, Vol. 52. S. Karger Publ., Basel, Switzerland.

European Council. 1988. Council Directive (EEC) No. 88/388 on the approximation of the laws of the Member States relating to flavor for use in foodstuffs and to source materials for their production. Off. J. Eur. Comm. L184:61-66.

Hazleton, L. W., H. K. Murer, R. Thiessen Jr., T. W. Tusing, and B. R. Zeitlin. 1956. Toxicity of coumarin. J. Pharmacol. Exp. Ther. 118:348-358.

Lavine, B. K., D. T. Corona, and U. D. N. T. Perera. 2012. Analysis of vanilla extract by reversed phase liquid chromatography using water rich mobile phases. Microchem. J. 103:49-61.

Niessen, W. M. A., P. Manini, and R. Andreoli. 2006. Matrix effects in quantitative pesticide analysis using liquid chromatography-mass spectrometry. Mass Spectrom. Rev. 25:881-899.

Ohashi, M., H. Omae, M. Hashida, Y. Sowa, and S. Imai. 2007. Determination of vanillin and related flavor compounds in cocoa drink by capillary electrophoresis. J. Chromatogr. A 1138:262-267.

Rahim, A. A., B. Saad, H. Osman, N. Hashim, S. Yahya, and K. M. Talib. 2011. Simultaneous determination of diethylene glycol, diethylene glycol monoethyl ether, coumarin and caffeine in food items by gas chromatography. Food Chem. 126:1412-1416.

Rychlik, M. 2008. Quantification of free coumarin and its liberation from glucosylated precursors by stable isotope dilution assays based on liquid chromatography-tandem mass spectrometric detection. J. Agric. Food Chem. 56:796-801.

Sola-Larrañaga, C., and I. Navarro-Blasco. 2009. Optimization of a slurry dispersion method for minerals and trace elements analysis in infant formulae by ICP OES and FAAS. Food Chem. 115:1048-1055.

Sproll, C., W. Ruge, C. Andlauer, R. Godelmann, and D. W. Lachenmeier. 2008. HPLC analysis and safety assessment of coumarin in foods. Food Chem. 109:462-469.

Xie, W., C. Han, Y. Qian, H. Ding, X. Chen, and J. Xi. 2011. Determination of neonicotinoid pesticides residues in agricultural samples by solid-phase extraction and liquid chromatography-tandem mass spectrometry. J. Chromatogr. A 1218:4426-4433. 